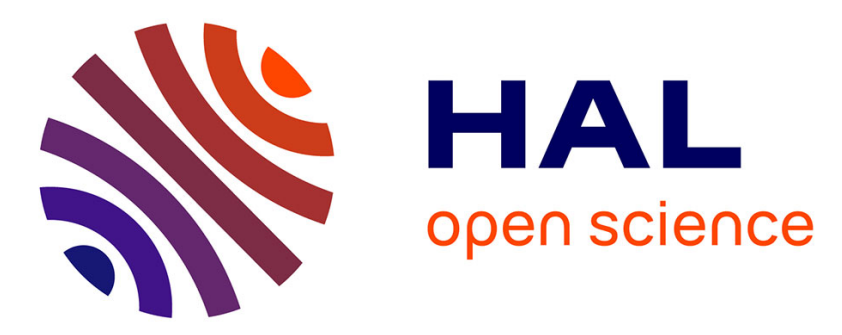

\title{
Tone mapped HDR images contrast enhancement using piecewise linear perceptual transformation
}

Ba Chien Thai, Anissa Mokraoui

\section{To cite this version:}

Ba Chien Thai, Anissa Mokraoui. Tone mapped HDR images contrast enhancement using piecewise linear perceptual transformation. EUSIPCO, Sep 2019, A Coruna, Spain. 10.23919/EUSIPCO.2019.8902728 . hal-02428241

\section{HAL Id: hal-02428241 \\ https://hal.science/hal-02428241}

Submitted on 5 Jan 2020

HAL is a multi-disciplinary open access archive for the deposit and dissemination of scientific research documents, whether they are published or not. The documents may come from teaching and research institutions in France or abroad, or from public or private research centers.
L'archive ouverte pluridisciplinaire HAL, est destinée au dépôt et à la diffusion de documents scientifiques de niveau recherche, publiés ou non, émanant des établissements d'enseignement et de recherche français ou étrangers, des laboratoires publics ou privés. 


\section{Tone mapped HDR images contrast enhancement using piecewise linear perceptual transformation}

\author{
Ba Chien Thai \\ University of Science and Technology \\ 54 Nguyen Luong Bang Street, Lien Chieu District \\ Danang, Vietnam \\ tbchien@dut.udn.vn
}

\author{
Anissa Mokraoui \\ L2TI, Institut Galilée, Université Paris 13 \\ 99 avenue Jean Baptiste Clément \\ Villetaneuse, France \\ anissa.mokraoui@univ-paris13.fr
}

\begin{abstract}
This paper addresses the conversion problem of High Dynamic Range (HDR) images into Low Dynamic Range (LDR) images. The aim of this conversion is to ensure a good visual rendering of the displayed Tone Mapped (TM) HDR images in accordance with the observers' assessment. To do so, the proposed algorithm adjusts a piecewise linear function to the logarithm luminance distribution of the HDR image to adapt this transformation to the perceptual quantizer in accordance with the Human Visual System (HVS). The computation of the slope value of the piecewise linear transformation is simplified. Indeed, it is given by the ratio between the probability and the logarithm luminance distance in the sampling bin. This leads to histograms which tend to be flat, thus inducing a contrast enhancement of the tone mapped HDR images in both under-exposed and overexposed areas. Simulation results provide good results, both in terms of visual quality and TMQI metric, compared to existing competitive TM approaches.
\end{abstract}

Index Terms-High Dynamic Range image, Low Dynamic Range image, Tone mapping, Histogram, Contrast enhancement.

\section{INTRODUCTION}

Acquiring visual information (e.g. images, videos) of real scenes representing the full range of luminance values of real world (i.e. including both under- and over-exposed areas) is possible through High Dynamic Range (HDR) capture devices. However, the cost of these devices remain too expensive. Therefore to continue viewing HDR content on conventional Low Dynamic Range (LDR) display devices, requires the reduction of the Dynamic Range (DR) of the HDR content using specific tone mapping algorithms. Indeed the main purpose of HDR image Tone Mapping (TM) approach is to find a trade-off between the relevant information (e.g. details, contrast, brightness...) to be preserved or discarded in the image so as to ensure a good visual quality of the displayed image on LDR devices that would be appreciated by observers.

A state of the art on HDR image TM approaches is fairly complete in [1], [2] and [3]. Among the developed TM strategies, this paper quickly reviews those that caught our attention because of their performance. Some approaches aim only at preserving detail or enhancing contrast, while others prefer to improve both. In what follows, the described approaches aimed to highlight mainly the details. A second generation of wavelets based on the edge content of the image avoiding having pixels from both sides of an edge has been proposed in In [4]. In [5], a separable non-linear multiresolution approach based on essentially non-oscillatory interpolation strategy has been developed. These approaches take into account the singularities (e.g. edge points) in their mathematical models preserving then the structural information of the HDR images. A non-separable version has been investigated and proposed in [6]. The results provided in [4], [5] and [6] show that the decomposition of the HDR image on different resolution levels would seem to be a good strategy. However, the choice of the decomposition filters is extremely important and decisive in the extractive power of the approximation and detail information.

However other TM strategies aimed both to preserve detail and enhance contrast. Indeed in [9], an edge-preserving bilateral filter is introduced to decompose the HDR image into two layers: a base layer encoding large-scale variations and a detail one. Contrast is then reduced only in the first layer while the details are kept unchanged. In [10], an adaptive logarithmic mapping method of luminance values is presented. It concerns the adjustment of the logarithmic basis depending on the radiance of the pixels. In [11], a subband architecture related on an oversampled Haar pyramid representation is proposed. Subband coefficients are re-scaled according to a gain control function reducing the high frequency magnitudes and boosting low ones.

Below are described methods that enhance contrast using in most cases histograms. In [12], a TM optimization approach using a histogram adjustment between linear mapping and the equalized histogram mapping is developed. A modification of this approach is made in [13]. The latter considers both histogram equalization and human sensitivity to the light function. A piecewise linear perceptual quantizer modeling the "s-shaped" curve of the Human Visual Adaptation with getting more optional contrast by changing the norm space is proposed in [7]. Later, in [8], authors proposed a combination of a near optimal lifting scheme and perceptual quantizer to enhance the contrast and preserve more details in the tone mapped HDR images.

This paper is organized as follows. Section II presents the the proposed TM HDR image approach. The later is based on the adjustment of the contrast according to a perceptual piecewise linear quantizer. Section III discusses the simulation 
results. Section IV concludes the paper.

\section{PROPOSED TONE MAPPING HDR IMAGE APPROACH}

The purpose of this section is to find the TM HDR values. For this, the proposed approach adjusts locally the distribution of the HDR image logarithm luminance values according to the HVS to enhance the contrast using a simplified piecewise linear function compared to [7].

The original HDR image is assumed to be of size $N \times M$. Denote $\widetilde{l}_{H D R}$ the HDR image luminance. It is considered in the logarithm domain since the latter is well adapted to the HVS. It is denoted $\mathbf{I}_{H D R}$ and defined as follows:

$$
\begin{array}{r}
\mathbf{I}_{H D R}:=\left\{\mathbf{I}_{H D R}\left(x_{n}, y_{m}\right)=\log _{10}\left(\widetilde{l}_{H D R}\left(x_{n}, y_{m}\right)\right)\right. \\
\text { for } 1 \leq n \leq N \text { and } 1 \leq m \leq M\},
\end{array}
$$

where $\mathbf{I}_{H D R}\left(x_{n}, y_{m}\right)$ is the HDR logarithm luminance value of the pixel located at position $\left(x_{n}, y_{m}\right)$ on the image.

\section{A. Non-uniform distribution of the HDR logarithm luminance}

The $\mathbf{I}_{H D R}$ values are first sorted and classified into equal $B$ bins defined by cutting points denoted $c_{u H D R}^{i}$ (with $1 \leq$ $i \leq B$ ). The index $i$ refers to the bin number. A nonuniform histogram equalization is also performed. $c_{n u H D R}^{i}$ (with $1 \leq i \leq B$ ) cutting points, defining the bounds of the non-uniform consecutive $B$ bins, are deduced. From uniform and non-uniform cutting points, new cutting points locally adapted to the logarithm luminance values are deduced as described below.

The new lower bound (i.e. new cutting point) of each bin, denoted $l_{H D R}^{i}(1)$, is then adjusted by the introduction of the parameter $\beta^{i}$ as follows:

$$
l_{H D R}^{i}(1)=c_{u H D R}^{i}+\beta_{i}\left(c_{n u H D R}^{i}-c_{u H D R}^{i}\right),
$$

where $\beta_{i}$ is a positive parameter smaller than 1 depending on the sub-interval $\left[c_{u H D R}^{i}, c_{n u H D R}^{i}\right]$. Since the lower cutting point $l_{H D R}^{i}(1)$ is deduced as the average of the logarithm luminance values on each sub-interval $\left[c_{u H D R}^{i}, c_{n u H D R}^{i}\right]$ as in [13], the parameter $\beta_{i}$ is then given by:

$$
\beta_{i}=\frac{\operatorname{mean}\left(\mathbf{I}_{H D R}\left[c_{u H D R}^{i}, c_{n u H D R}^{i}\right]\right)-c_{u H D R}^{i}}{c_{n u H D R}^{i}-c_{u H D R}^{i}} .
$$

Note that this strategy avoids to get empty bins. The $\mathbf{I}_{H D R}$ values are then classified into non-uniform $B$ bins as follows:

$$
\begin{array}{r}
\mathbf{I}_{H D R}=\left\{l_{H D R}(k) \text { for } k=1, \ldots, N \times M\right\}= \\
\left\{\left[l_{H D R}^{1}(1), \ldots, l_{H D R}^{1}\left(K_{1}\right)\right], \ldots,\left[l_{H D R}^{i}(1), \ldots, l_{H D R}^{i}\left(K_{i}\right)\right],\right. \\
\left.\ldots,\left[l_{H D R}^{B}(1), \ldots, l_{H D R}^{B}\left(K_{B}\right)\right]\right\},
\end{array}
$$

depending on the quantization level set, where $K_{i}$ is the number of values in the $i$-th bin (i.e. $1 \leq i \leq B ; K_{i}>0$ ) and satisfying the following relation $\sum_{i=1}^{B} K_{i}=N \times M$.

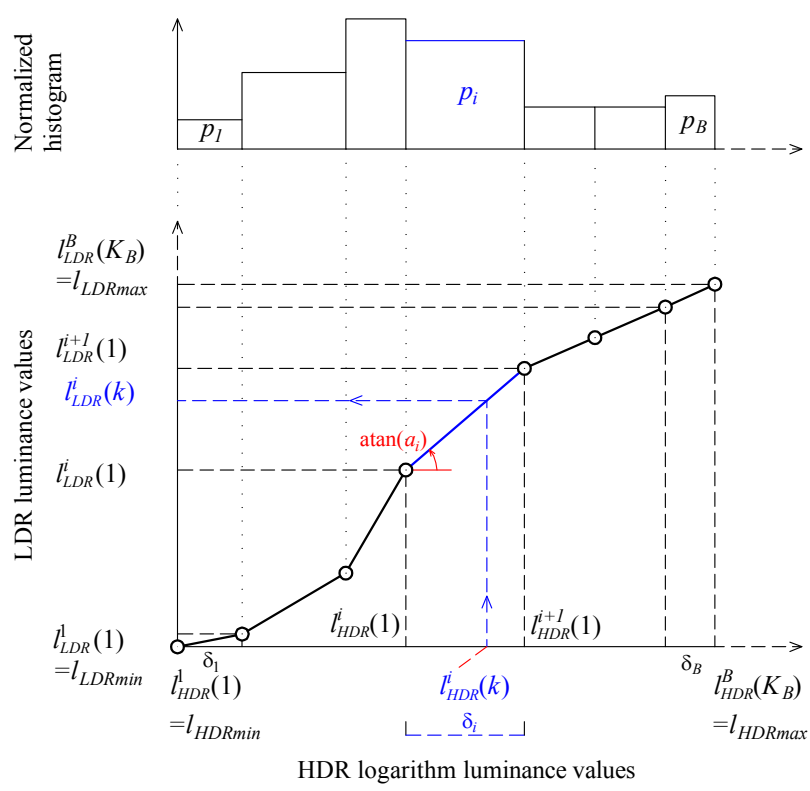

Fig. 1: TM approach using piecewise linear curve.

\section{B. Piecewise linear perceptual quantizer}

The "s-shaped" TM perceptual curve, as discussed in [15] and [16], is modelled by a piecewise linear curve on each bin (see Fig. 1). Consider the $i$-th bin, defined by $\left[l_{H D R}^{i}(1), \ldots, l_{H D R}^{i}\left(K_{i}\right)\right]$, the TM values are then modeled as follows:

$$
l_{L D R}^{i}(k)=a_{i} l_{H D R}^{i}(k)+b_{i} \text { with } k \in\left[1, K_{i}\right],
$$

where $a_{i}$ (with $a_{i} \neq 0$ ) and $b_{i}$ are two unknown parameters depending on the $i$-th bin.

Introduce $\delta_{i}$ as the difference between HDR luminance in two consecutive bins (see Fig.1):

$$
\delta_{i}=l_{H D R}^{i+1}(1)-l_{H D R}^{i}(1) .
$$

Denote $l_{L D R \max }$ (respectively $l_{L D R \min }$ ) the maximum (respectively minimum) LDR luminance value. The LDR dynamic range (i.e. $\left.l_{L D R \max }-l_{L D R \min }\right)$ must satisfy the following relation:

$$
l_{L D R \max }-l_{L D R \min }=\sum_{i=1}^{B} a_{i} \cdot \delta_{i} .
$$

The unknown slope $a_{i}$ has been computed in a sophisticated way in [7]. In this paper, a simplified solution is proposed. Remember that the slope $a_{i}$ is proportional to $\left(l_{L D R}^{i}(k)-\right.$ $\left.l_{L D R}^{i}(1)\right)$ which is also unknown. The latter can be linked to the probability $p_{i}$ of the $l_{H D R}^{i}(k)$ value in the $i$-th bin and is given by $p_{i}=\frac{K_{i}}{\sum_{i=1}^{B} K_{i}}$. The probability associated to the LDR dynamic range (i.e. $\left.l_{L D R \max }-l_{L D R \min }\right)$ is assumed to be equal to one. Therefore one can deduce that $l_{L D R}^{i}(k)-$ $l_{L D R}^{i}(1)=p_{i} \times\left(l_{L D R \max }-l_{L D R \min }\right)$. The slope $a_{i}$ is then computed as follows:

$$
a^{i}=\frac{l_{L D R \max }-l_{L D R \min }}{\delta_{i}} \cdot p_{i} .
$$


The more probability $p_{i}$, the more value $a_{i}$, and vice versa. The set of slopes satisfy the constraint condition given by (8).

The unknown parameter $b_{i}$ is computed by the following formula: $b_{i}=l_{L D R}^{i}(1)-a_{i} \times l_{H D R}^{i}(1)$. The HDR mapped values are deduced according to equation (6). The global piecewise linear curve is continuous and strictly monotonic increasing according to the positive slopes (i.e $a_{i}>0$, or angles $\left.0^{\circ}<\operatorname{atan}\left(a_{i}\right)<90^{\circ}\right)$.

\section{Simulation RESUlts}

This section discusses the performance of the proposed TM HDR image approach. The TM image objective quality is measured with the "Tone-Mapped image Quality Index" (TMQI) metric [21]. Simulations have been conducted under Matlab environnement using the HDR Toolbox ([1]) with 274 test HDR images. For lack of space, we only present the results obtained with 8 HDR images ("Bottle Small", "Office", "Oxford Church", "Memorial", "Light", "ClarkCenter", "DollDoll", "ClockBuilding", "AtriumNight" and "PeaceRocks") having different dynamic range (or contrast ratio) from $8 \mathrm{f}$ stops to 29 f-stops.

The results are compared to: (i) CEDP [8] with norm 1 and level 1; (ii) NUHA [7] with norm 1; (iii) NONSEP ENOCA [6]; (iv) SEP ENO-CA [5] with parameters $\alpha_{1}=0.3$, $\alpha_{2}=0.7$; (v) Li TMO [11] with Haar multiscale; (vi) Fattal [4] using RBW method with parameters $\alpha=0.8$, $\beta=0.3, \gamma=0.8$; (vii) Duan [12] using $\beta=0.5$; (viii) TMOs in HDR Toolbox: Drago [10], Reinhard [17], Ward [18], Durand [9], Schlick [20] using default parameters as given in the HDR Toolbox. The parameters are chosen so as to give the best results in terms of TMQI metric in all methods.

Table I provides the TMQI metrics. The proposed TM approach namely "Proposed" is deployed with $B=256$, $l_{L D R \max }=255$ and $l_{L D R \min }=0$. The results show that our TM approach is competitive to those developed in the literature. Fig. 2 provides :(i) the TM "ClarkCenter" image of good visual quality in adequacy with the TMQI metric; (ii) normalized histogram with flatness; and (iii) tone mapping curve closest to the "s-shaped" curve.

TABLE I: Tone Mapped Image Quality Index (TMQI)

\begin{tabular}{lclllll}
\hline TMOs & Anturium & Bottle & Office & Church & Memorial & Light \\
DR f-stops & 8.73 & 16.03 & 16.29 & 15.46 & 18.38 & 17.46 \\
\hline Drago [10] & 0.874 & 0.801 & 0.800 & 0.814 & 0.800 & 0.800 \\
Reinhard [17] & 0.778 & 0.807 & 0.826 & 0.789 & 0.791 & 0.794 \\
Ward [18] & 0.806 & 0.783 & 0.775 & 0.817 & 0.795 & 0.789 \\
Durand [9] & 0.811 & 0.892 & 0.825 & 0.929 & 0.814 & 0.800 \\
Tumblin [19] & 0.715 & 0.713 & 0.735 & 0.675 & 0.759 & 0.750 \\
Schlick [20] & 0.770 & 0.835 & 0.926 & 0.970 & 0.787 & 0.780 \\
Duan [12] & $\mathbf{0 . 9 6 4}$ & 0.916 & $\mathbf{0 . 9 5 5}$ & $\mathbf{0 . 9 8 6}$ & 0.935 & 0.916 \\
Fattal [4] & 0.889 & 0.928 & 0.943 & 0.889 & 0.927 & $\mathbf{0 . 9 7 1}$ \\
Li [11] & $\mathbf{0 . 9 6 4}$ & $\mathbf{0 . 9 5 4}$ & 0.854 & 0.877 & 0.834 & 0.888 \\
Anas [13] & 0.900 & 0.837 & 0.896 & 0.850 & 0.823 & 0.832 \\
SEPENO [5] & 0.896 & 0.934 & 0.943 & 0.895 & 0.932 & 0.970 \\
NONSEP [6] & 0.938 & 0.873 & 0.935 & 0.820 & 0.832 & 0.932 \\
NUHA [7] & 0.929 & 0.850 & 0.927 & 0.897 & 0.922 & 0.916 \\
CEDP [8] & 0.957 & 0.878 & 0.943 & 0.980 & $\mathbf{0 . 9 6 0}$ & $\mathbf{0 . 9 7 1}$ \\
\hline Proposed & $\mathbf{0 . 9 7 5}$ & $\mathbf{0 . 9 5 9}$ & $\mathbf{0 . 9 6 0}$ & $\mathbf{0 . 9 8 7}$ & $\mathbf{0 . 9 6 1}$ & $\mathbf{0 . 9 8 5}$ \\
\hline
\end{tabular}

Fig. 3 compares the visual quality of the TM "AtriumNight" images using "Li" and our approach. Although "Li" approach added some post-processing to improve the visual rendering such as cutting off the brightest and darkest parts as well as adding $15 \%$ of a histogram equalized layer to the result, our approach is of better visual quality and higher TMQI. Fig. 4 concerns the TM "DollDoll" images using "Fattal" and our approach. These images confirm the performance of our proposal.

The visual quality of the TM "ClockBuilding" images, given by Fig. 5, using "SEPENO" and our approach show that some details are lost on "SEPENO" TM mapped image compared to our approach. Moreover, our TM image is of better contrast. A similar result is provided by Fig. 6 where the "PeaceRock" HDR image has been mapped using "NUHA" and our method. One can observe that the brightness of our TM image is better.

For TM "church" images in Fig. 7, although TMQI metric is slightly different for "Duan" method (0.986) and our approach (0.987), the visual rendering of our TM image is better.

The performance of our approach is confirmed on more than 274 test HDR images where the details and contrast are better represented than other competitive methods for a wide range of f-stops.

\section{CONCLUSION}

This paper proposed a simplified piecewise linear perceptual quantizer for tone mapped HDR images contrast enhancement. The HDR logarithm luminance values distribution are adjusted to fit the perceptual "s-shape" curve. Simulation results confirm the relevance of the proposed approach both in terms of the TMQI objective metric and the visual quality of the displayed image.

\section{REFERENCES}

[1] Banterle, F., Artusi, A., Debattista, K., and Chalmers, A., Advanced High Dynamic Range Imaging: Theory and Practice, AK Peters (now CRC Press), ISBN: 978-156881-719-4 (2011).

[2] Dufaux, F., Le Callet, P., Mantiuk, R., Mrak, M., High Dynamic Range Video 1st Edition : From Acquisition, to Display and Applications, ISBN: 9780081004128 (April 2016)

[3] Reinhard, E., Heidrich, Wolfgang., Debevec, Paul., Pattanaik, S., Ward, G., and Myszkowski, K., High Dynamic Range Imaging 2nd Edition: Acquisition, Display, and Image-Based Lighting, ISBN: 9780123749147 (May 2010)

[4] Fattal, R., Edge-Avoiding Wavelets and their Applications, ACM Trans. Graph (2009).

[5] Thai, B.C., Mokraoui, A., and Matei, B., Performance Evaluation of High Dynamic Range Image Tone Mapping Operators Based on Separable Non-linear Multiresolution Families, 24th European Signal Processing Conference, pp. 1891-1895 (August 2016).

[6] Thai, B.C., Mokraoui, A., and Matei, B., Image Tone Mapping Approach Using Essentially Non-Oscillatory Bi-quadratic Interpolations Combined with a Weighting Coefficients Strategy, 17th IEEE International Symposium on Signal Processing and Information Technology, ISSPIT (December 2017).

[7] Thai, B.C., Mokraoui, A., and Matei, B., Piecewise linear perceptual quantizer as a non-uniform histogram equalization adjustment for contrast enhancement of tone mapped HDR images, 9th International Symposium on Signal, Image, Video and Communications, ISIVC (November 2018).

[8] Thai, B.C., Mokraoui, A., and Matei, B., Contrast Enhancement and Details Preservation of Tone Mapped High Dynamic Range Images, Journal of Visual Communication and Image Representation, (2018).

[9] Durand, F., and Dorsey, J., Fast Bilateral Filtering for The Display of High-dynamic-range Images, ACM Transactions on Graphics (TOG) Proceedings of ACM SIGGRAPH 21, pp. 257-266 (2002). 


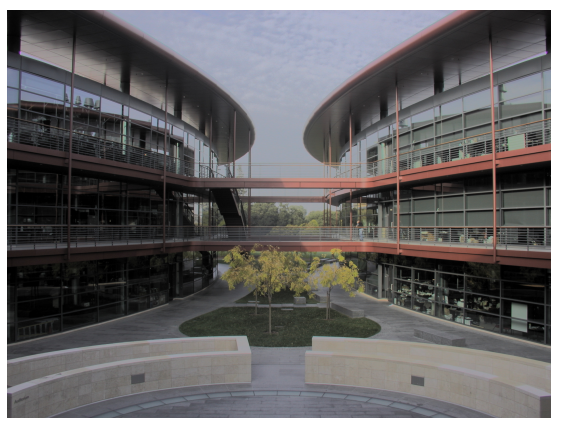

(a) Tone mapped image (TMQI=0.945)

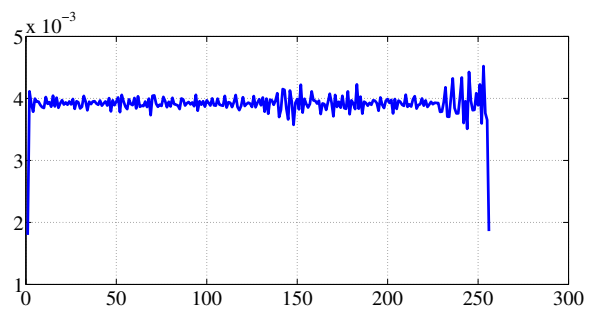

(b) Normalized histogram

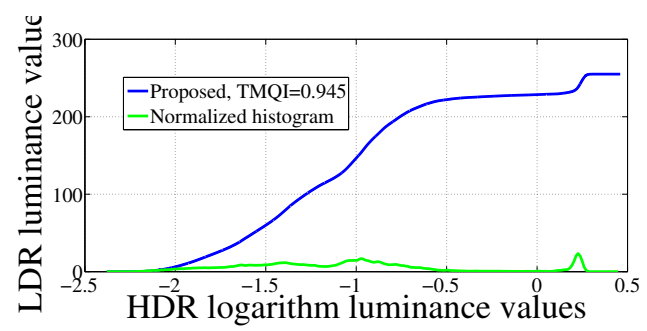

(c) Tone mapping curve

Fig. 2: TM "ClarkCenter" image (19.42 f-stops).

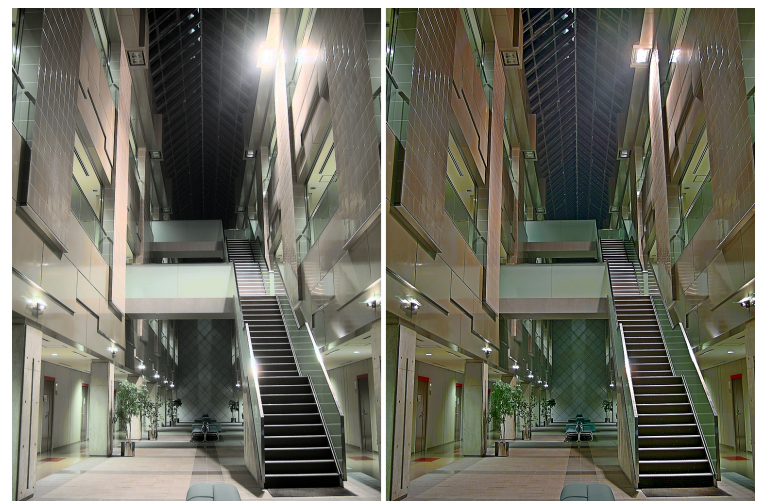

Fig. 3: TM "AtriumNight" image (28.68 f-stops): (Left) Proposed (TMQI=0.958), (Right) Li (TMQI=0.888).

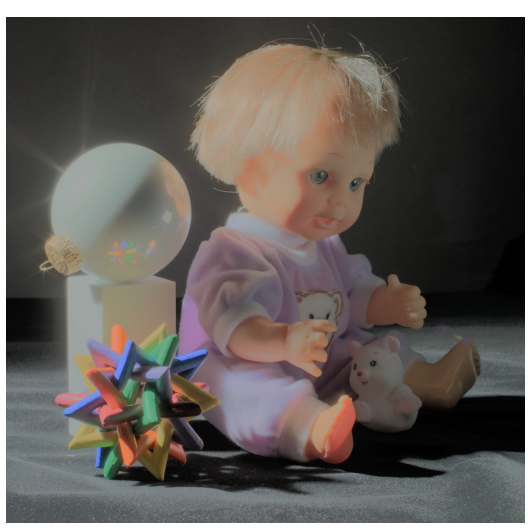

(a) Proposed (TMQI $=0.888$ )

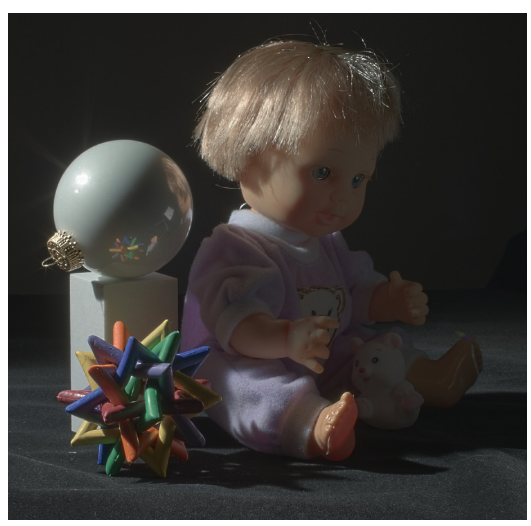

(b) Fattal RBW (TMQI=0.733)

Fig. 4: TM ”DollDoll" image (13.89 f-stops).

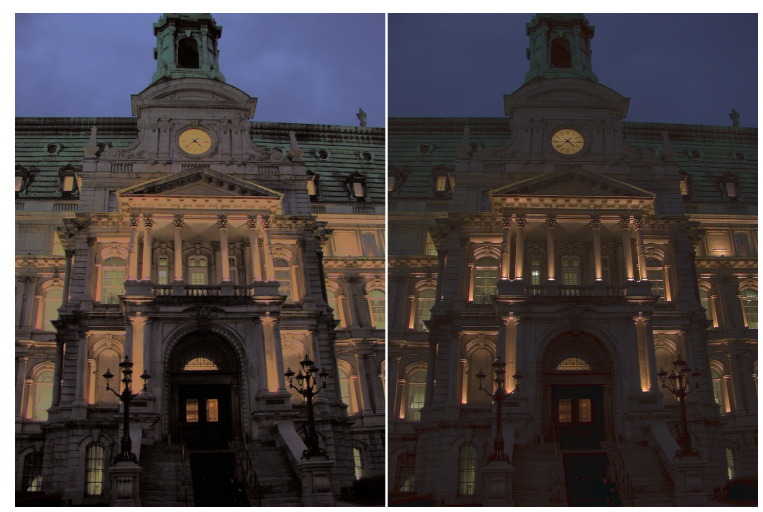

Fig. 5: TM "ClockBuilding" image (14.19 f-stops): (Left) Proposed (TMQI=0.962), (Right) SEPENO (TMQI=0.753). 


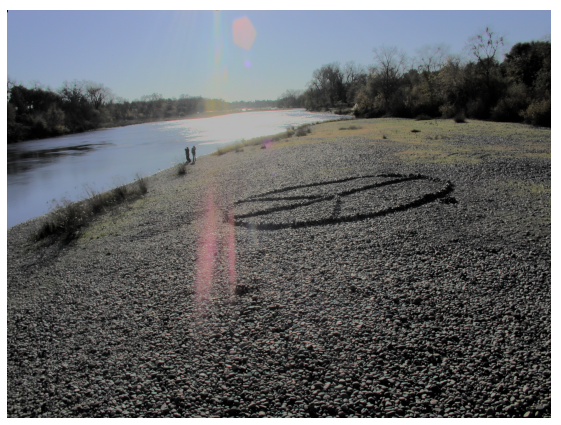

(a) Proposed (TMQI=0.893)

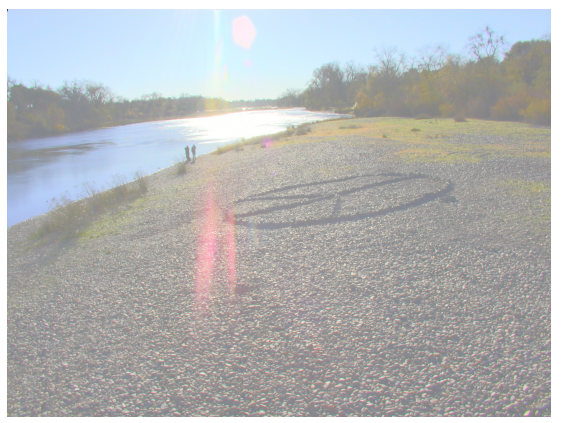

(b) NUHA Norm 1 (TMQI=0.865)

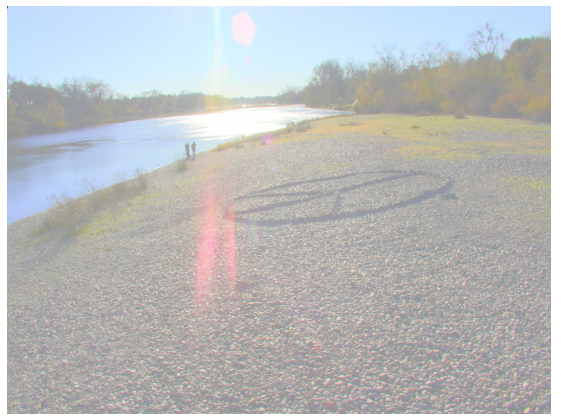

(c) NUHA Norm 2 (TMQI=0.771)

Fig. 6: TM "PeaceRocks" image (24.14 f-stops).

[10] Drago, F., Myszkowski, K., Annen, T., and Chiba, N., Adaptive Logarithmic Mapping for Displaying High Contrast Scenes, Computer Graphics Forum 22, pp. 419-426 (2003).

[11] Li, Y., Sharan, L., and Adelson, E.H., Compressing and Companding High Dynamic Range Images with Subband Architectures, ACM Trans. Graph. 24, pp. 836-844 (July 2005).

[12] Duan, J., Bressan, M., Dance, C., and Qiu, G., Tone-mapping High Dynamic Range Images by Novel Histogram Adjustment, Pattern Recognition, vol. 43, pp. 1847-1862 (2010).

[13] Husseis, A., Mokraoui, A., and Matei, B., Revisited Histogram Equalization as HDR Images Tone Mapping Operators, 17th IEEE International Symposium on Signal Processing and Information Technology, ISSPIT (December 2017).

[14] Mai, Z., Mansour, H., Nasiopoulos, P., and Ward, R., Visually-favorable tone-mapping with high compression performance, Image Processing (ICIP) 17th IEEE International Conference on, pp. 1285-1288, ISSN

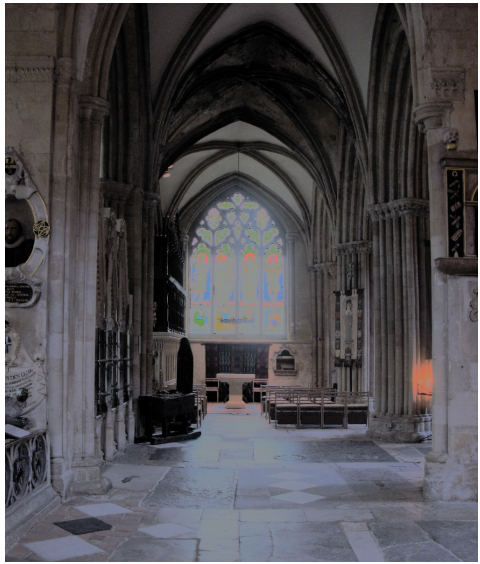

(a) Proposed $(\mathrm{TMQI}=0.987)$

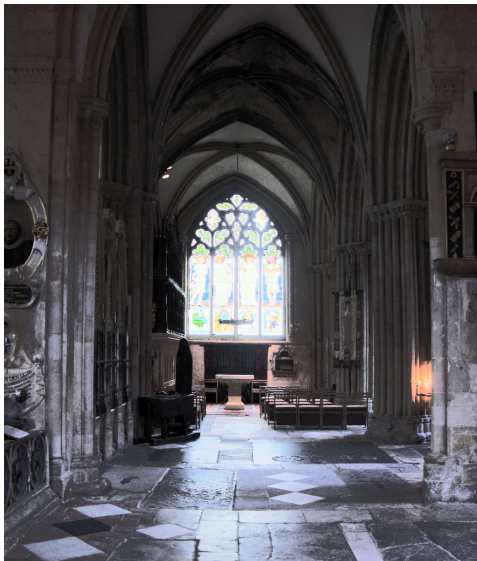

(b) Duan $(\mathrm{TMQI}=0.986)$

Fig. 7: TM "Church" image (15.46 f-stops).

1522-4880 (2010).

[15] Dowling, J. E., The Retina: An Approachable Part of the Brain, Cambridge, Belknap Press (1987).

[16] Geisler, W. S., Effects of Bleaching and Backgrounds on the Flash Response of the Cone System, Journal of Physiology, 312:413-434 (1981).

[17] Reinhard, E., and Devlin, K., Dynamic Range Reduction Inspired by Photoreceptor Physiology, IEEE Transactions on Visualization and Computer Graphics 11, pp. 13-24 (2005).

[18] Ward, G., Rushmeier, H., and Piatko, C., A Visibility Matching Tone Reproduction Operator for High Dynamic Range Scenes, IEEE Transactions on Visualization and Computer Graphics 3, pp. 291-306 (1997).

[19] Tumblin, J., and Rushmeier, H., Tone Reproduction for Realistic Images, IEEE Comput. Graph. Appl, pp. $42-48$ (1993).

[20] Schlick, C., Quantization Techniques for Visualization of High Dynamic Range Pictures, In Proceeding of the Fifth Eurographics Workshop on Rendering, pp. 7-18 (1994).

[21] Yeganeh, H. and Wang, Z., Objective Quality Assessment of Tonemapped Images, IEEE Trans. on Image Processing, vol. 22, pp. 657-667 (February 2013). 